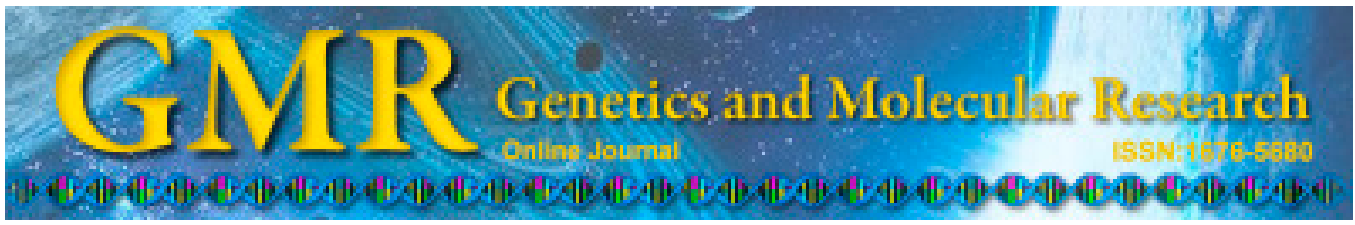

\title{
Effect of Xin Mai Jia on atherosclerosis in rats
}

\author{
P. Li ${ }^{1}$, G.P. Pan ${ }^{1}$, M. Jia ${ }^{3}$, Q.Q. Wang ${ }^{1}$, Z.G. Guo ${ }^{4}$, F.R. Zhao ${ }^{1}$, G.L. Lei ${ }^{4}$, \\ G.R. Wan ${ }^{5}$ and G.M. Wan ${ }^{6}$ \\ ${ }^{1}$ College of Pharmacy, Xinxiang Medical University, Xinxiang, China \\ ${ }^{2}$ Research Center for Immunology, Department of Laboratory Medicine, \\ Xinxiang Medical University, Xinxiang, China \\ ${ }^{3}$ Puyang City Health School, Puyang, Henan, China \\ ${ }^{4}$ Modern Technology Education Center, Xinxiang Medical University, \\ Xinxiang, China \\ ${ }^{5}$ School of Basic Medical Sciences, Xinxiang Medical University, Xinxiang, \\ China \\ ${ }^{6}$ The First Affiliated Hospital, ZhengZhou University, Zhengzhou, China
}

Corresponding author: G.R. Wan

E-mail: guangruiwandoc@126.com

Genet. Mol. Res. 14 (2): 6018-6027 (2015)

Received September 17, 2014

Accepted February 26, 2015

Published June 1, 2015

DOI http://dx.doi.org/10.4238/2015.June.1.19

ABSTRACT. We investigated the therapeutic effect of Xin Mai Jia
(XMJ) on atherosclerosis (AS) in rats. Rat models of AS were estab-
lished by peritoneally injecting vitamin D, feeding a high-fat diet, and
inducing balloon injuries in rats. The stomachs of the rats were irrigated
continuously for 10 weeks with XMJ. Blood lipid- and hemorheology-
related indices of blood samples were detected. Pathological changes
in the right common carotid arterial tissues were also determined. The
protein expression levels of endothelial nitric oxide synthase, angio-
tensin-1, and endothelin-1 were determined by western blotting. XMJ
reduced cholesterol, trigylecride, and low-density lipoprotein levels as
well as blood viscosity, sedimentation, and hematocrit. Furthermore,
XMJ alleviated vascular endothelial injury and reduced/eliminated
atherosclerotic plaques. In contrast, XMJ significantly increased the
endothelium-dependent relaxing response of the AS rat models. The 
western blotting results showed that XMJ upregulated endothelial nitric oxide synthase but downregulated angiotensin-1 and endothelin-1. $\mathrm{XMJ}$ prevented the development of AS by regulating blood lipid levels, hemorheology, and vascular function.

Key words: Angiotensin-1; Atherosclerosis; Vascular function; Endothelial nitric oxide synthase; Endothelin-1

\section{INTRODUCTION}

Atherosclerosis (AS) is an important arteriosclerotic cardiovascular disease. AS lesions originate from the endarterium. In general, symptoms of AS include hypertension, hypercholesterolemia, or diabetes. AS is one of the leading causes of death in developed countries (Linsel-Nitschke and Tall, 2005; Hansson, 2005). However, the pathogenesis of AS is not fully understood, and many risk factors lead to AS. Studies have reported that AS is caused by the co-actions of vascular endothelial lesions, inflammation, and immunological dysfunction.

Pathological studies have shown that AS related to phlegm syndrome can be treated with traditional Chinese medicine. Researchers have explored the mechanism by which AS is prevented by eliminating phlegm and resolving stasis theory using traditional Chinese medicine. Xin Mai Jia (XMJ) is composed of astaxanthin, functional red rice, pueraria isoflavone, soybean isoflavone, bamboo leaf flavones, and resveratrol (Patent No. ZL 20101 0536001.x). XMJ can alleviate the symptoms of AS and reduce or eliminate complications after few months; moreover, XMJ can reduce blood lipids, normalize blood pressure, and improve sleep quality. However, the mechanism by which XMJ exerts its therapeutic effects remains unclear. In the present study, XMJ was used to treat rats with AS, after which we determined the changes in blood lipids, hemorheology, endothelial nitric oxide synthase (eNOS), angiotensin-1 (AT-1), and endothelin-1 (ET-1) levels to explore the possible mechanism.

\section{MATERIAL AND METHODS}

\section{Animals}

Forty-eight male Sprague-Dawley (SD) rats weighing 200-300 g (Laboratory Animal Center of HeNan Province) were used as subjects. All rats were fed with diets and water. This study was carried out in strict accordance with the recommendations in the Guide for the Care and Use of Laboratory Animals of the National Institutes of Health. The animal use protocol was reviewed and approved by the Institutional Animal Care and Use Committee of Xinxiang Medical University.

\section{Groups}

The SD rats were randomly divided into 8 groups: control group; drug-medium control group; model group; $6 \mathrm{mg} \cdot \mathrm{kg}^{-1} \mathrm{day}^{-1}$ lovastatin treatment group; $0.7813 \mathrm{~g} \cdot \mathrm{kg}^{-1} \mathrm{day}^{-1} \mathrm{Zhi}$ Bituo treatment group (Chengdu Di'ao Group); $0.5934 \mathrm{~g} \cdot \mathrm{kg}^{-1} \mathrm{day}^{-1}$ low-dose XMJ treatment group; $1.875 \mathrm{~g} \cdot \mathrm{kg}^{-1} \mathrm{day}^{-1}$ middle-dose XMJ treatment group; and $5.925 \mathrm{~g} \cdot \mathrm{kg}^{-1} \mathrm{day}^{-1}$ high-dose XMJ treatment group. 


\section{AS model}

AS rat models were established by feeding high-fat diets, injecting vitamin D3, and inducing balloon injuries in all groups except the normal control group and the positive control group, which were fed basic diets. High-fat diets included $81.5 \%$ basic diets, $10 \%$ lard, $0.5 \%$ sodium cholate, $3 \%$ cholesterol, and $5 \%$ sugar. The dose of high-fat diets was $150 \mathrm{~g} /$ day. Common carotid arterial intima injury was induced after the rats were fed for 4 weeks. The rats were continuously fed with high-fat diets for 10 weeks.

\section{Pathomorphological observation}

After the rats were anesthetized, the common carotid artery was obtained and embedded in paraffin. Paraffin sections were then prepared by dehydration, transparency, wax dip, and embedment. The sections were then stained with hematoxylin and eosin and observed under an electron microscope.

\section{Blood fat detection}

Approximately, $2 \mathrm{~mL}$ blood was extracted from the common carotid artery and centrifuged at $3000 \mathrm{rpm}, 730 \mathrm{~g}$ for $15 \mathrm{~min}$ at $4^{\circ} \mathrm{C}$. The supernatant was obtained, and the levels of total cholesterol, triglyceride (TG), high-density lipoprotein (HDL), and low-density lipoprotein (LDL) were detected using the oxidase reaction.

\section{Hemorheology test}

Blood viscosity was tested using an LG-R-80F automatic blood rheometer (Beijing gtmsteellex Science Instrument Co., Ltd., Beijing, China).

\section{Vascular endothelial function detection}

One part of the common carotid artery was cut to form a vascular circle and then treated with $10^{-6} \mathrm{M}$ norepinephrine to reach the largest shrink tension. Approximately $10^{-8}-10^{-4}$ $\mathrm{M}$ acetylcholine was added. We recorded vascular tension and observed the effect of the drug on the endothelial-dependent relaxation response.

\section{Western blot}

Approximately, $100 \mathrm{mg}$ common carotid artery was collected, and $0.5 \mathrm{~mL}$ cold lysis buffer solution was added (lysate: inhibitor phosphatase $=1: 5,1 \mu \mathrm{L}$ protease inhibitor and 5 $\mu \mathrm{L} 100 \mathrm{mM}$ phenylmethylsulfonyl fluoride). The resulting solution was homogenized in ice water and centrifuged at $1000 \mathrm{rpm}$ for $5 \mathrm{~min}$ at $4^{\circ} \mathrm{C}$. The supernatant contained the total protein extract. Protein concentration was evaluated using the bicinchoninic acid method. Protein samples were stored at $-80^{\circ} \mathrm{C}$. The lysates $(50 \mu \mathrm{g}$ protein/well) were electrophoresed by sodium dodecyl sulfate-polyacrylamide gel electrophoresis for $4 \mathrm{~h}$ and then blotted onto polyvinylidene fluoride membranes. The blots were subsequently incubated with primary antibodies overnight at $4^{\circ} \mathrm{C}$, washed, and incubated for $1.5 \mathrm{~h}$ with horseradish peroxidase-conjugated 
secondary antibodies at 1:4000 dilutions. After washing, we developed the membranes using an electrochemiluminescence system. Protein expression levels were determined by analyzing the signals captured on the membranes by using the Image-Pro Plus 6.0 analyzer (Media Cybernetics, Inc., Rockville, MD, USA) with $\beta$-actin as an internal control.

\section{Statistical analysis}

Statistical analysis was performed using the SPSS 13.0 software (SPSS, Inc., Chicago, IL, USA), and all results are reported as mean \pm standard deviation. One-way analysis of variance was performed to compare different groups. Statistical significance was considered to be $\mathrm{P}<0.05$.

\section{RESULTS}

\section{Pathomorphological changes}

Compared with the control group, the model group exhibited evident endothelial injury as observed under electron microscope. Yellow atherosclerotic plaques were also observed in the model group. XMJ reduced the degree of endothelial injury and promoted plaque disappearance in a dose-dependent manner (Figure 1). Electron microscopy revealed that the integrity of the vascular endothelial cell membrane was retained and no special particles were observed in the nucleus, and a significant difference was observed between the control group and the model group (Figure 2).

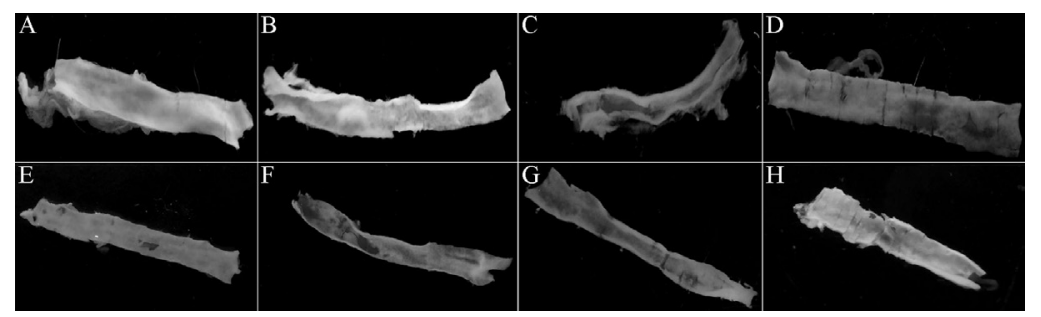

Figure 1. Observation of common carotid artery with eyes. A. Normal control group. B. Drug-medium control group. C. Lovastatin group. D. Zhibituo group. E. Model group. F. Low-dose Chinese medicine group. G. Mediumdose Chinese medicine group. H. High-dose Chinese medicine group.

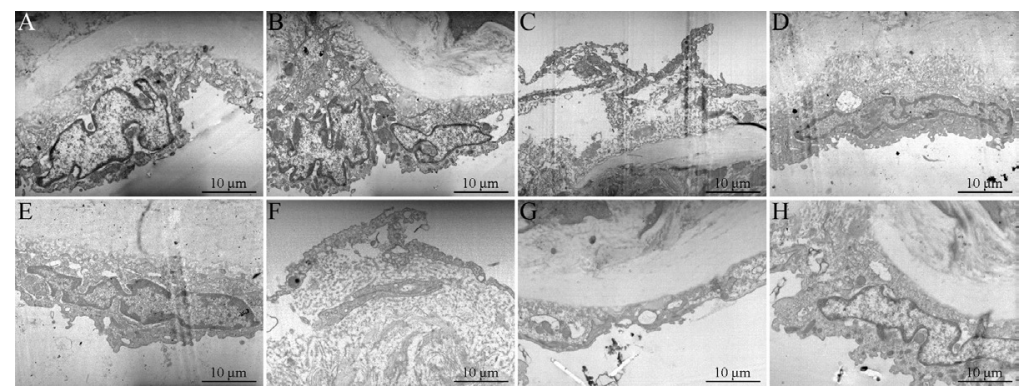

Figure 2. Observation of endothelial cells in the common carotid artery of rats using light microscope (1000X). A. Normal control group. B. Drug-medium control group. C. Model group. D. Lovastatin group. E. Zhibituo group. F. Low-dose Chinese medicine group. G. Medium-dose Chinese medicine group. H. High-dose Chinese medicine group. 


\section{Blood fat}

XMJ significantly reduced cholesterol, TG, and LDL levels, but increased glutamic acid, HDL, and apolipoprotein levels in a dose-dependent manner $(\mathrm{P}<0.05$, Table 1$)$ compared with the control group.

\begin{tabular}{|c|c|c|c|c|c|c|c|c|}
\hline Group/index & GLU & $\mathrm{CHOL}$ & TG & HDL & LDL & $\mathrm{HDL} / \mathrm{CHOL}$ & APOA-1 & APOB \\
\hline Control & $15.11 \pm 0.65^{* \#}$ & $0.72 \pm 0.09^{* \#}$ & $1.13 \pm 0.09^{* \#}$ & $0.42 \pm 0.08^{* \#}$ & $0.13 \pm 0.03 * \#$ & $0.51 \pm 0.07^{* \#}$ & $0.17 \pm 0.04^{* \#}$ & $0.13 \pm 0.03^{* \#}$ \\
\hline Drug-medium control & $11.85 \pm 0.54^{* \#}$ & $0.54 \pm 0.07 * \#$ & $0.42 \pm 0.04 * \#$ & $0.47 \pm 0.07^{* \#}$ & $0.11 \pm 0.02 * \#$ & $0.56 \pm 0.08^{* \#}$ & $0.18 \pm 0.05^{* \#}$ & $0.11 \pm 0.02 * \#$ \\
\hline Model & $5.95 \pm 0.44^{\# \Delta}$ & $1.13 \pm 0.16^{\# \Delta}$ & $1.82 \pm 0.12^{\# \Delta}$ & $0.27 \pm 0.05^{\# \Delta}$ & $0.28 \pm 0.05^{\# \Delta}$ & $0.34 \pm 0.06^{\# \Delta}$ & $0.07 \pm 0.01^{\# \Delta}$ & $0.02 \pm 0.00^{\# \Delta}$ \\
\hline Lovastatin & $6.82 \pm 0.52^{* \| \Delta}$ & $0.51 \pm 0.06^{* \# \Delta}$ & $0.48 \pm 0.07^{* \# \Delta}$ & $0.33 \pm 0.06^{* H \Delta}$ & $0.22 \pm 0.04^{* * \Delta}$ & $0.43 \pm 0.05^{* \# \Delta}$ & $0.08 \pm 0.01^{* \# \Delta}$ & $0.08 \pm 0.01^{* \# \Delta}$ \\
\hline Zhibituo & $6.95 \pm 0.62 * \# \Delta$ & $0.55 \pm 0.08^{* \# \Delta}$ & $0.58 \pm 0.08^{* \# \Delta}$ & $0.35 \pm 0.06^{* \# \Delta}$ & $0.23 \pm 0.03^{* \# \Delta}$ & $0.48 \pm 0.04^{* \# \Delta}$ & $0.09 \pm 0.02 * \# \Delta$ & $0.06 \pm 0.01^{* \# \Delta}$ \\
\hline $\begin{array}{l}\text { Low-dose Chinese } \\
\text { medicine }\end{array}$ & $5.54 \pm 0.47^{* \# \Delta}$ & $0.48 \pm 0.06^{* * \Delta}$ & $0.97 \pm 0.07^{* * \Delta}$ & $0.39 \pm 0.06^{* \# \Delta}$ & $0.19 \pm 0.05^{* \# \Delta}$ & $0.44 \pm 0.05^{* \# \Delta}$ & $0.14 \pm 0.03 *{ }^{* \Delta}$ & $0.05 \pm 0.01 * * \Delta$ \\
\hline $\begin{array}{l}\text { Medium-dose } \\
\text { Chinese medicine }\end{array}$ & $8.44 \pm 0.81^{* * \Delta}$ & $0.44 \pm 0.06^{* * \Delta \Delta}$ & $0.84 \pm 0.06^{* * \Delta}$ & $0.41 \pm 0.07^{* \# \Delta}$ & $0.15 \pm 0.03^{* \# \Delta}$ & $0.38 \pm 0.04^{* * \Delta}$ & $0.09 \pm 0.01^{* \# \Delta}$ & $0.09 \pm 0.02^{* * \Delta}$ \\
\hline High-dose Chinese & $14.23 \pm 0.76^{* \Delta}$ & $0.55 \pm 0.07^{* \Delta}$ & $0.99 \pm 0.12^{* \Delta}$ & $0.32 \pm 0.04^{* \Delta}$ & $0.16 \pm 0.02 * \Delta$ & $0.41 \pm 0.06^{* \Delta}$ & $0.11 \pm 0.02^{* \Delta}$ & $0.11 \pm 0.03^{* \Delta}$ \\
\hline
\end{tabular}

$* \mathrm{P}<0.05$ vs the model group; ${ }^{*} \mathrm{P}<0.05$ vs the high-dose Chinese medicine group; $\Delta \mathrm{P}<0.05$ vs the normal control group.

\section{Hemorheology}

Hemorheology results indicated that XMJ reduced the vascular viscosity of rat blood plasma in a dose-dependent manner. A significant difference was observed between the control and model groups $(\mathrm{P}<0.05$, Table 2$)$.

Table 2. Viscosity of rat blood plasma $(\mathrm{N}=6$, means $\pm \mathrm{SD})$.

\begin{tabular}{lccrr}
\hline Group/shear rate & 200 & 30 & 3 & 1 \\
\hline Control & $3.12 \pm 0.34^{* \#}$ & $4.43 \pm 0.35^{* \#}$ & $7.44 \pm 0.54^{* \#}$ & $12.43 \pm 1.33^{* \#}$ \\
Drug-medium control & $3.17 \pm 0.18^{* \#}$ & $3.93 \pm 0.08^{* \#}$ & $7.29 \pm 0.73^{* \#}$ & $12.07 \pm 1.95^{* \#}$ \\
Model group & $4.24 \pm 0.91^{\# \Delta}$ & $5.45 \pm 1.08^{\# \Delta}$ & $10.94 \pm 1.80^{\# \Delta}$ & $18.92 \pm 2.75^{\# \Delta}$ \\
Lovastatin & $3.42 \pm 0.30^{* \# \Delta}$ & $4.34 \pm 0.10^{* \# \Delta}$ & $8.51 \pm 1.02^{* \# \Delta}$ & $14.56 \pm 2.91^{* * \Delta}$ \\
Zhibituo & $3.08 \pm 0.28^{* \# \Delta}$ & $3.93 \pm 0.26^{* \# \Delta}$ & $7.73 \pm 0.45^{* \# \Delta}$ & $13.23 \pm 1.14^{* * \Delta}$ \\
Low-dose Chinese medicine & $3.27 \pm 0.38^{* \# \Delta}$ & $4.12 \pm 0.36^{* \# \Delta}$ & $7.89 \pm 0.31^{* \# \Delta}$ & $13.28 \pm 0.65^{* * \Delta}$ \\
Medium-dose Chinese medicine & $2.74 \pm 0.26^{* \# \Delta}$ & $3.24 \pm 0.29^{* \# \Delta}$ & $5.34 \pm 0.49^{* \# \Delta}$ & $8.18 \pm 0.86^{* * \Delta}$ \\
High-dose Chinese & $3.16 \pm 0.47^{* \Delta}$ & $3.85 \pm 0.51^{* \Delta}$ & $6.81 \pm 0.67^{* \Delta}$ & $10.92 \pm 0.83^{* \Delta}$ \\
\hline
\end{tabular}

${ }^{*} \mathrm{P}<0.05$ vs the model group; ${ }^{*} \mathrm{P}<0.05$ vs the high-dose Chinese medicine group; ${ }^{\wedge} \mathrm{P}<0.05$ vs the normal control group.

\section{Vascular function}

XMJ increased endothelial-dependent relaxation in a dose-dependent manner. A significant difference was detected between the control and model groups $(\mathrm{P}<0.05$, Figure 3$)$.

\section{Western blot}

Western blot results showed that the expression of eNOS significantly reduced in the model group compared to that in the control group $(\mathrm{P}<0.05)$. However, the expression levels of AT-1 and ET-1 were significantly higher $(\mathrm{P}<0.05)$ in the control group than that in the model group. XMJ increased the expression of eNOS and decreased the expression levels of AT-1 and ET-1. A significant difference was observed between the model group and the XMJ treatment group $(\mathrm{P}<0.05$, Figures 4-6). 


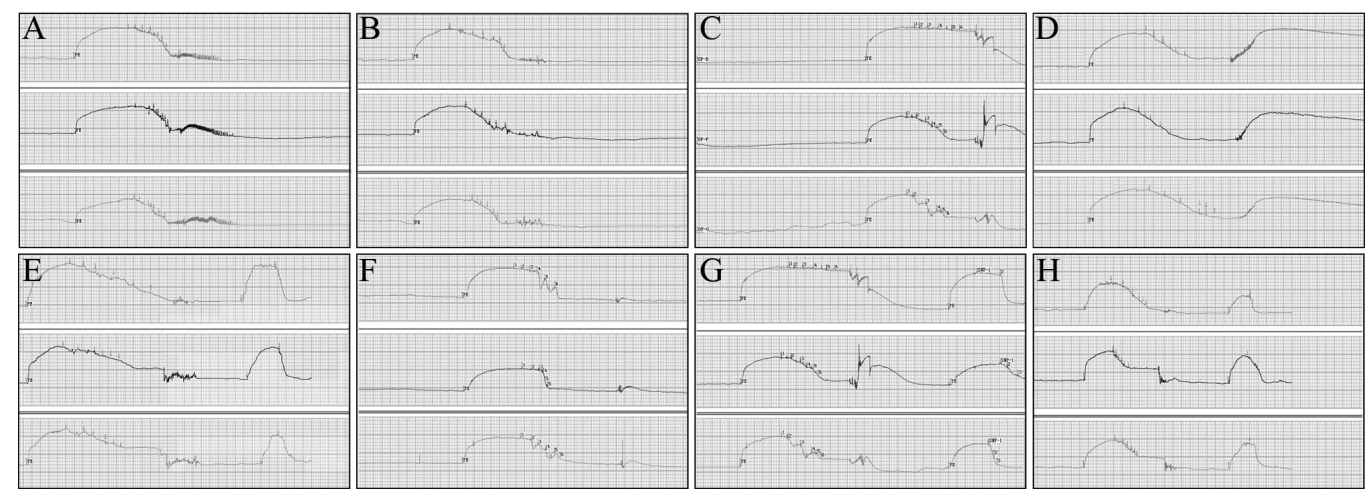

Figure 3. Endothelium-dependent reaction of the common carotid artery of rats $(\mathrm{N}=6$, means $\pm \mathrm{SD})$. A. Normal control group. B. Drug-medium control group. C. Model group. D. Lovastatin group. E. Zhibituo group. F. Lowdose Chinese medicine group. G. Medium-dose Chinese medicine group. H. High-dose Chinese medicine group.

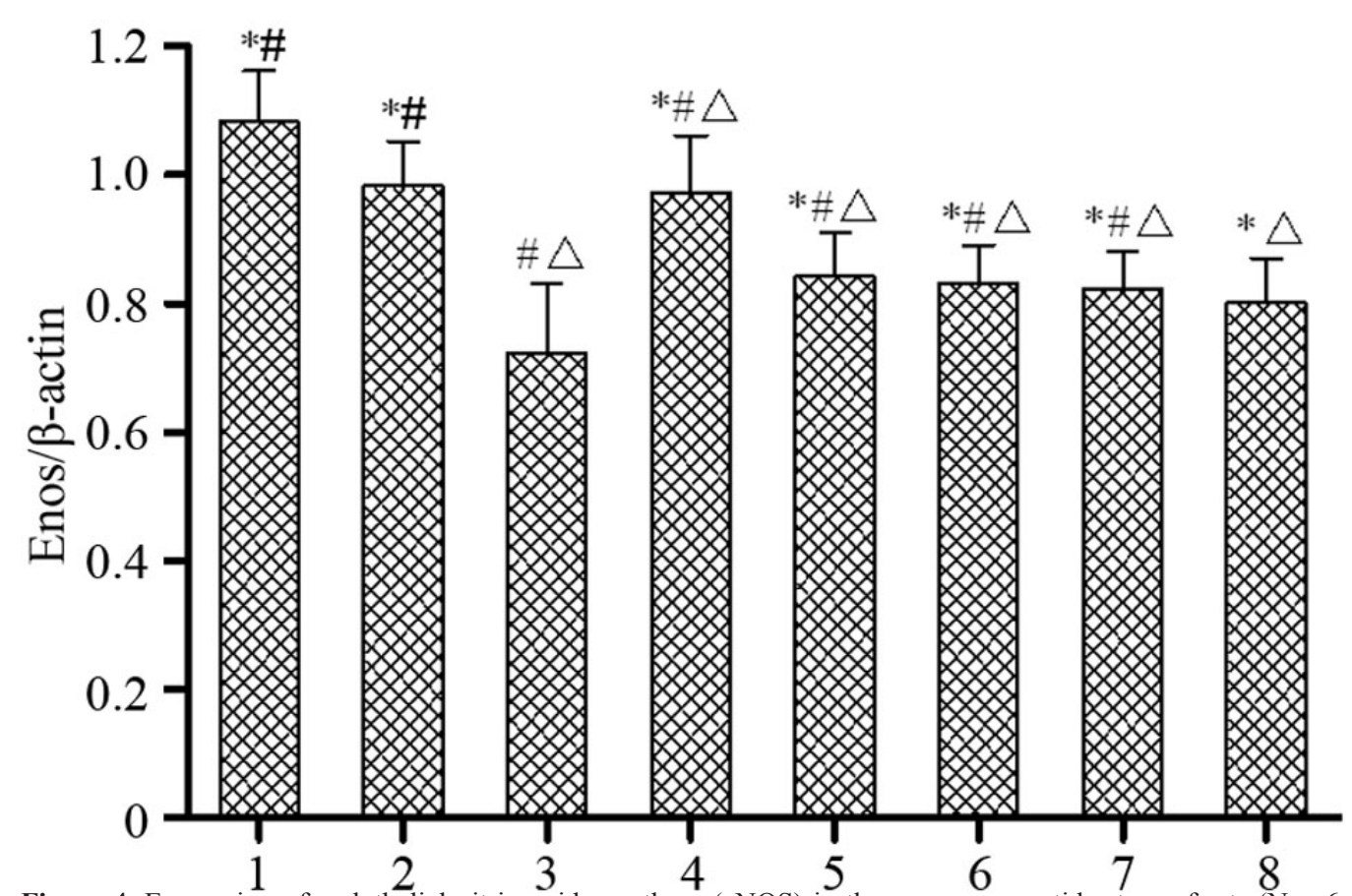

Figure 4. Expression of endothelial nitric oxide synthase (eNOS) in the common carotid artery of rats $(\mathrm{N}=6$, means $\pm \mathrm{SD}$ ). Column 1: Normal control group. Column 2: Drug-medium control group. Column 3: Model group. Column 4: Lovastatin group. Column 5: Zhibituo group. Column 6: Low-dose Chinese medicine group. Column 7: Medium-dose Chinese medicine group. Column 8: High-dose Chinese medicine group. $\left({ }^{*} \mathrm{P}<0.05 v s\right.$ the model group; ${ }^{\mathrm{P}}<0.05$ vs the high-dose Chinese medicine group; ${ }^{\triangle} \mathrm{P}<0.05$ vs the normal control group). 


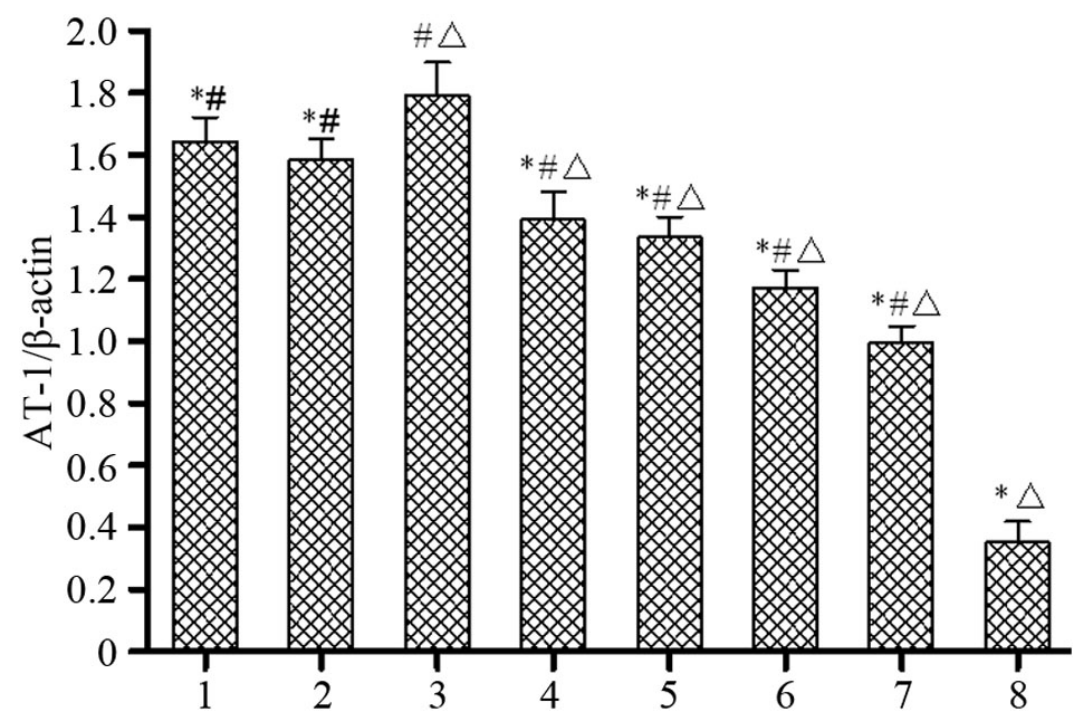

Figure 5. Expression of angiotensin-1 (AT-1) in the common carotid artery of rats $(\mathrm{N}=6$, means $\pm \mathrm{SD})$. Column 1: Normal control group. Column 2: Drug-medium control group. Column 3: Model group. Column 4: Lovastatin group. Column 5: Zhibituo group. Column 6: Low-dose Chinese medicine group. Column 7: Medium-dose Chinese medicine group. Column 8: High-dose Chinese medicine group. $\left({ }^{*} \mathrm{P}<0.05\right.$ vs the model group; ${ }^{*} \mathrm{P}<0.05$ vs the high-dose Chinese medicine group; ${ }^{\wedge} \mathrm{P}<0.05$ vs the normal control group).

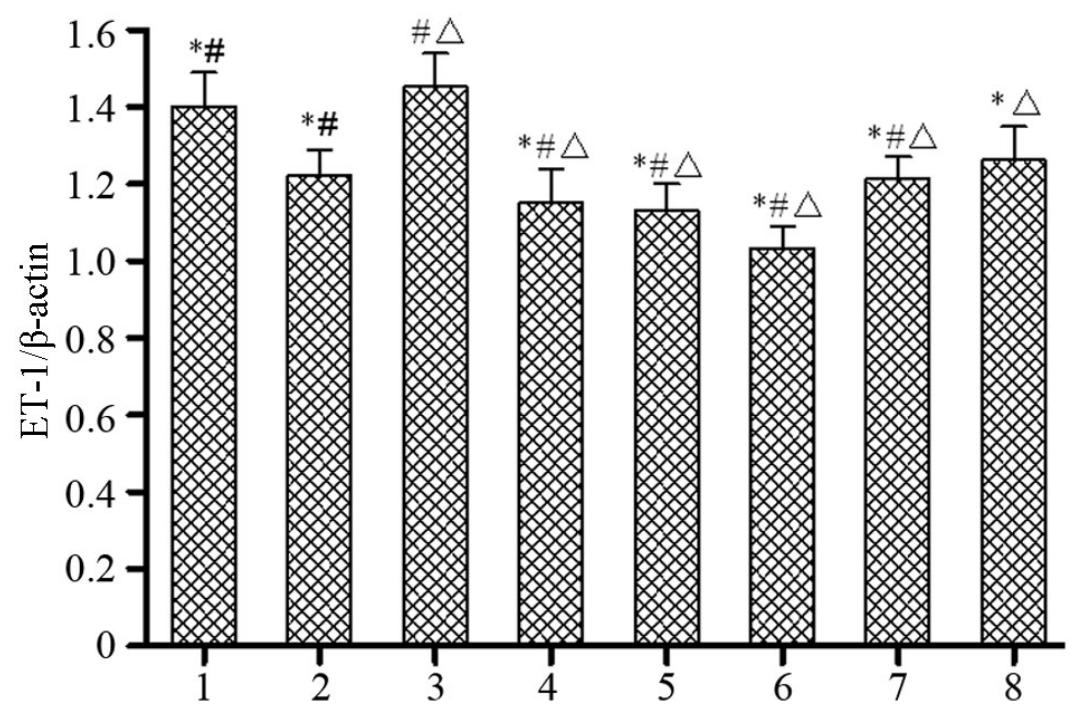

Figure 6. Expression of endothelin-1 (ET-1) in the common carotid artery of rats $(\mathrm{N}=6$, means $\pm \mathrm{SD})$. Column 1: Normal control group. Column 2: Drug-medium control group. Column 3: Model group. Column 4: Lovastatin group. Column 5: Zhibituo group. Column 6: Low-dose Chinese medicine group. Column 7: Medium-dose Chinese medicine group. Column 8: High-dose Chinese medicine group. $\left({ }^{*} \mathrm{P}<0.05\right.$ vs the model group; ${ }^{*} \mathrm{P}<0.05$ vs the high-dose Chinese medicine group; ${ }^{\triangle} \mathrm{P}<0.05$ vs the normal control group). 


\section{DISCUSSION}

AS is an important pathological basis for cardio-cerebral vascular diseases. This condition is also considered to be a target for exploring treatment measures for cardiovascular and cerebrovascular diseases and studying the mechanisms of cardio-cerebral vascular diseases. Atherogenesis is the developmental process of atheromatous plaques. Numerous risk factors lead to AS, and high blood lipid levels and hemorheology promote AS. Other studies have reported that hyperlipidemia is an independent risk factor for AS and cardio-cerebral vascular diseases (Nordestgaard et al., 2010; Zurek et al., 2013). Fatty deposits damage the endothelium in the artery by triggering dyslipidemia, thereby upregulating the expression of cytokines to form foam cells. Foam cells and platelets encourage the migration and proliferation of smooth muscle cells and macrophages, which in turn ingest lipids, are replaced with collagen, and transform into foam cells. Epidemiological research confirmed that hypertriglyceridemia is a risk factor of AS. AS is positively related to total cholesterol, TG, and LDL levels and is considered to be an independent risk factor of coronary heart disease. However, HDL directly or indirectly transfers cholesterol to the liver to decrease cholesterol deposits in the artery wall. The results of the present study indicated that XMJ reduced total cholesterol, TG, and LDL levels, but increased HDL level, indicating that XMJ promoted blood fat reduction.

Hyperlipidemia is the pathological basis of AS and causes changes in hemorheology. Therefore, AS may be prevented or treated by understanding hemorheological changes. Studies have demonstrated that blood fat and raises blood viscosity are closely related to AS (Ruggiero et al., 2013) Our results showed that XMJ effectively treated AS by reducing blood fat and viscosity.

Vascular endangium lesions caused lipid deposits to reduce arterial elasticity, after which the narrow cavity induced cardiovascular symptoms (Dong et al., 2012). Thus, lipid deposits may be reduced and vascular elasticity can be recovered. In the present study, XMJ significantly increased the endothelium-dependent relaxation of the model rats $(\mathrm{P}<0.05)$ in a dose-dependent manner. This result indicated that XMJ could be used to treat AS and improved vascular elasticity.

The mechanism by which XMJ improved vascular elasticity should be further examined. AS represents endothelium dysfunction, including nitric oxide (NO) dyssynthesis mediated by the endothelium, which is due to the disequilibrium of ET and NO. The expression levels of eNOS, ET-1, and AT-1 were detected by Western blotting. NO is catalyzed by NOS in order to bring about vascular relaxation response. The biological effect of NO can be observed through the study of NOS, since NO cannot be easily measured. Under physiological conditions, NO is generated by eNOS in the vascular system. NOS can also extend the blood vessel, regulate blood pressure, inhibit platelet aggregation (Huang et al., 2006), prevent smooth muscle cell proliferation, and suppress adhesion between endothelial cells and monocytes (Mujynya-Ludunge et al., 2005; Gkaliagkousi and Ferro, 2011). Moreover, eNOS, a key enzyme in the NO/cyclic guanosine monophosphate signal pathway, regulates vascular tone and protects endothelial cell function by coupling $\mathrm{Ca}^{2+}$ and calmodulin. NO is catalyzed and released by eNOS to enter near smooth muscle cells and activate guanylate cyclase, which catalyzes cyclic guanosine monophosphate to activate the calcium pump to reduce intracellular free calcium and promote smooth muscle relaxation. Cyclic guanosine monophosphate also promotes smooth muscle relaxation by inhibiting protein kinase activity (Murad, 2006). Studies have shown that NO not only functions as a vascular relaxing factor, but also maintains 
vascular wall structure (Ozüm et al., 2008). Low NOS expression reduces NO levels. NOS antagonists significantly accelerated AS development in some animal experiments (Zancan et al., 1999; Sweazea and Walker, 2011). In the present study, eNOS expression decreased in the AS model group. This result suggested that NO reduction is crucial in AS development. XMJ appeared to increase eNOS expression in model rats.

ETs are proteins that constrict blood vessels and increase blood pressure (Yanagisawa et al., 1988). These proteins are released by injured vascular endothelial cells. ET level is positively correlated with the vascular lesion counts of AS (Nakaki et al., 1989; Lerman et al., 1991). Studies have found that high plasma ET levels are related to vascular cell lesions (Naruse et al., 1991; Ray et al., 1993) during AS development. Reriani et al. (2010) also confirmed that an ET-1 receptor antagonist significantly improved the endothelial function of patients with coronary AS, showing that endogenous ET is important in early atherogenesis. Angiotensin II is an important molecule in the rennin-angiotensin system and increases blood pressure. Early studies reported the presence of a positive feedback control mechanism between ET and angiotensin II (Emori et al., 1989; Dohi et al., 1999). Thus, both ET and angiotensin II can function as markers to evaluate the degree of vascular lesion. In the present study, the expression levels of ET-1 and AT-1 were significantly higher in the AS model group than that in the control group.

Low biological NO activity causes vasoconstriction and dysfunction of the endothelium by increasing ET expression (Bourque et al., 2011). An imbalance between NO and ET destroys the arterial endothelium and causes endothelial dysfunction. Furthermore, the most important and earliest characteristic of endothelial dysfunction is the loss of vascular endothelium-dependent function, which was observed in our study. XMJ appeared to increase eNOS expression and decrease ET-1 and AT-1 expression levels. The balance between NO and ET was then recovered, which may be a mechanism for treating AS.

\section{ACKNOWLEDGMENTS}

Research supported by major research projects of the Department of Science and Technology of Henan Province of China (\#121100910300).

\section{REFERENCES}

Bourque SL, Davidge ST and Adams MA (2011). The interaction between endothelin-1 and nitric oxide in the vasculature: new perspectives. Am. J. Physiol. Regul. Integr. Comp. Physiol. 300: R1288-R1295.

Dohi Y, Hahn AW, Boulanger CM, Bühler FR, et al. (1999). Endothelin stimulated by angiotensin II augments contractility of spontaneously hypertensive rat resistance arteries. Hypertension 19: 131-137.

Dong QS, Shang HT, Wu W, Chen FL, et al. (2012). Prefabrication of axial vascularized tissue engineering coral bone by an arteriovenous loop: a better model. Mater Sci. Eng. C. Mater Biol. Appl. 1; 32:1536-1541.

Emori T, Hirata Y, Ohta K, Shichiri M, et al. (1989). Secretary mechanism of immunoreactive endothelin in cultured bovine endothelial cells. Biochem. Biophsiol. Res. Commun. 160: 93-100.

Gkaliagkousi E and Ferro A (2011). Nitric oxide signalling in the regulation of cardiovascular and platelet function. Front. Biosci. 16: 1873-1897.

Hansson GK (2005). Inflammation, atherosclerosis, and coronary artery disease. N. Engl. J. Med. 352: 1685-1695.

Huang SS, Wei FC and Hung LM (2006). Ischemic preconditioning attenuates postischemic leukocyte - endothelial cell interactions: role of nitric oxide and protein kinase C. Circ. J. 70: 1070-1075.

Lerman A, Edwards BS, Hallett JW, Heublein DM, et al. (1991). Circulating and tissue endothelin immunoreactivity in advanced atherosclerosis. N. Engl. J. Med. 325: 997-1001.

Linsel-Nitschke P and Tall AR (2005). HDL as a target in the treatment of atherosclerotic cardiovascular disease. Nat. 
Rev. Drug. Discov. 4: 193-206.

Mujynya-Ludunge K, Viswambharan H, Driscoll R, Ming XF, et al. (2005). Endothelial nitric oxide synthase gene transfer restores endothelium-dependent relaxations and attenuates lesion formation in carotid arteries in apolipoprotein E-deficient mice. Basic Res. Cardiol. 100: 102-111.

Murad F (2006). Shattuck Lecture. Nitric oxide and cyclic GMP in cell signaling and drug development. N. Engl. J. Med. 355: 2003-2011.

Nakaki T, Nakayama M, Yamamoto S and Kato R (1989). Endothelin-mediated stimulation of DNA synthesis in vascular smooth muscle. Biochem. Biophys. Res. Commun. 158: 380-383.

Naruse M, Kawana M, Hifumi S, Naruse K, et al. (1991). Plasma immunoreactive endothelin, but not thrombomodulin, is increased in patients with essential hypertension and ischemic heart disease. J. Cardiovasc. Pharmacol. 17: S471-S474.

Nordestgaard BG, Chapman MJ, Ray K, Borén J, et al. (2010). Lipoprotein(a) as a cardiovascular risk factor: current status. Eur. Heart J. 31: 2844-2853.

Ozüm U, Bolat N, Gül E and Ozdemir O (2008). Endothelial nitric oxide synthase gene [G894T] polymorphism as a possible risk factor in aneurysmal subarachnoid hemorrhage. Acta Neurochir. (Wien) 150: 57-61.

Ray SG, McMurray JJ, Morton JJ and Dargie HJ (1993). Circulating endothelin in acute ischaemic syndromes. Br. Heart J. 67: 383-386.

Reriani M, Raichlin E, Prasad A, Mathew V, et al. (2010). Long-term administration of endothelin receptor antagonist improves coronary endothelial function in patients with early atherosclerosis. Circulation 122: 958-966.

Ruggiero D, Paolillo S, Ratta GD, Mariniello A, et al. (2013). Endothelial function as a marker of pre-clinical atherosclerosis:assessment techniques and clinical implications. Monaldi Arch Chest Dis. 80:106-110.

Sweazea KL and Walker BR (2011). High fat feeding impairs endothelin-1 mediated vasoconstriction through increased iNOS-derived nitric oxide. Horm. Metab. Res. 43: 470-476.

Yanagisawa M, Kurihara H, Kimura S, Tomobe Y, et al. (1988). A novel potent vasoconstrictor peptide produced by vascular cells. Nature 332: 411-415.

Zancan V, Santagati S, Bolego C, Vegeto E, et al. (1999). 17 Beta-estradiol decreases nitric oxide synthase II synthesis in vascular smooth muscle cells. Endocrinology 140: 2004-2009.

Zurek P, Dębiński M, Czerwiński W, Kondys M, et al. (2013). Acute myocardial infarction with simultaneous occlusions of two coronary arteries in a 44 year-old man. Kardiol. Pol. 71: 279-282. 